\title{
PATRONATO NACIONAL DE LA INFANCIA
}

\section{Labor del Servicio Social}

\author{
Por la Visitadoca Jefe, señorita ELENA HENRIQUEZ
}

Me es grato presentar, una vez más, a la consideración del Honorable Directorio del Patronato Nacional de la Infancia el trabajo efectuado durante el año 1939, por el Servicio Social de la Institución,

Como en años anteriores, el trabajo se desarrolló en forma normal; las $\mathrm{Visitadoras} \mathrm{Sociales} \mathrm{demostraron} \mathrm{especial} \mathrm{es-}$ mero en atender a las familias inscritas, aportando junto con sus conocimientos profesionales una abnegación a toda prueba. Debo dejar constancia que la labor de la Visitadora se hizo en estrecha colaboración con la dirección y el cuerpo médico de cada Gota de Leche. De esta manera se confirman las grandes ventajas del trabajo médico-social coordinado, que se realiza por un personal especializado. Las Directoras han ayudado a solucionar aquellos problemas económicos que pueden ser la causa básica del malestar de los asistidos. El Servicio Médico ha contribuído a resolver los problemas de su especialidad en forma eficaz.

Durante el curso del año esta Inspección tuvo serias dificultades para estabilizar al personal de Visitadoras en las Gotas de Leche, el que después de una corta estado en el $\mathrm{Pa}$ tronato pasaba a otros servicios con mayor renta y mejores perspectivas para el futuro.

Por otra pdrte, la experiencia ha demostrado que es superior el rendimiento de una Visitadora Social que conoce el - barrio en que actúa, el personal que colabora con ella y los recursos y medios de vida de los asistidos confiados a su cuidado.

Por las razones enunciadas, se procuró por todos los medios posibles que la Visitadora Social quedara largo tiempo en el Servicio, acordándose en agosto de 1939 fusionar algunos Servicios, tomando dos Gotas de Leche una sola Visi- 
tadora, secundada por una ayudante que desempeña la labor de secretaría de ofícina y que realiza los pequeños trámites que se le encomiendan. El resultado de este acuerdo, puesto en práctica en las Gotas de Leche "Guillermo Edwards Garriga", "José Abelardo Núñez" y "Luis Barros Valdés" que funcionan con doble turno: en "Manuel Guzmán Montt", que unió su servicio con "Mercedes y Teresa Lazcano" y "Luisa Vigil" con "Magdalena Valdés", ha correspondido a lo que se esperaba. Se ha considerado como un ascenso el servicio doble. constituyendo un aliciente para la Visita'dora Socixi que aspire a mayor renta dentro del mismo Patronato, cuando reúne las condiciones necesarias de eficiencia.

Gráficos: La semana de la Gota de Leche continuó desarrollándose en forma regular. El día señalado para la visita oficial de los miembros del Directorio las Visitadoras presentaron. en gráficos, el estado económico-sociàl de sus asistidos. Este trabajo, que significa un mayor esfuerzo dentro de la diaria labor que desempeñan, ha sido dẹbidamente apreciado por el señor Presidente del Patronato, a quien me hago un deber de mänifestar en notribre de las Visitadoras $y$ en el propio, nuestros agradecimientos por su palabras de estímulo y aliento que nos hacen desarrollar con mayor agrado nuestra labor dentro de la Institución.

Estudiados todó los datos recogidos de las 17. Gotas de Leche, exponemos el gráfico N. ${ }^{9} 1$ referente a 1 a constitución. de 3,179 hogares.

En primer término, podemos ver que el porcentaje de las uniones legalizadas civil y religiosamente ha disminuido en un $2,70 \%$. Por otro lado, las uniones libres demuestran un aumento de $3,30 \%$ disminuyendo paralelamente el de las madres solteras en $2,40 \%$. Diversas son las causas que han hecho disminuir el porcentaje de hogares legítimos: una de ellas, que a nuestro juicio es la principal, es el total desconocimiento que tienen nuestro pueblo de los beneficios que le rerorta la Ley del Matrimonio Civil. especialmente para los hijos, y la poca importancia que le dan al matrimonio religioso. Otra de las causas de la disminución del porcentaje de hogares legalmente constituídos es el ingreso reciente de rumerosas famílias que no habian sido atendidas con anterioridad por el Servicio Social del Patronato y en las que no se ha podido hacer todavía una labor social en ese sentido. El porcentaje más bajo de las madres solteras que aparece en el gráfico se debe a que éstas trabajan en su gran mayoría y sé acogen 


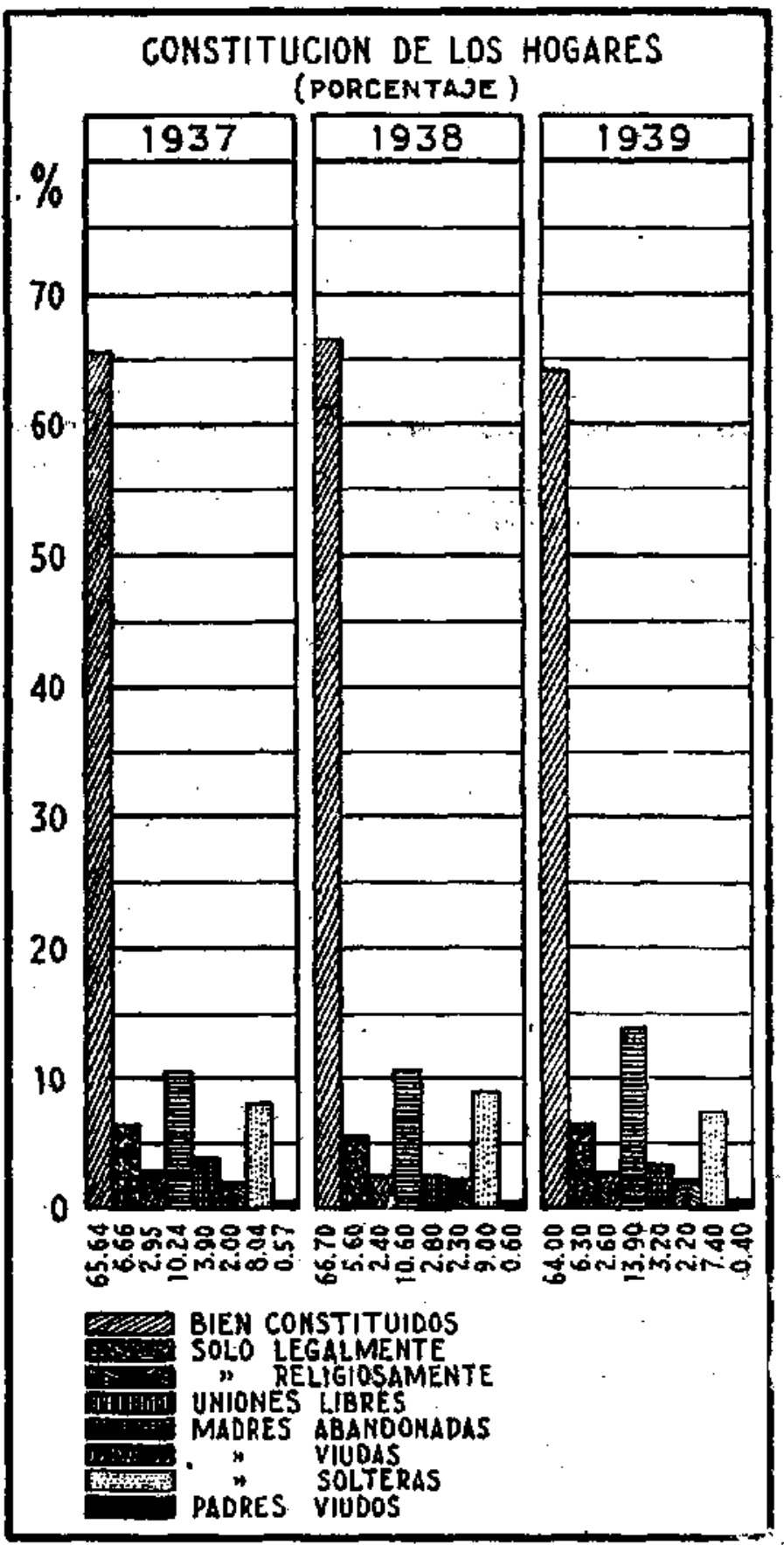


a los beneficios de la Ley 4,054. El Patronato sólo atiende a las madres verdaderamente indigentes y que no encuentran asistencia en ninguna otra parte. Lo mismo podemos decir de los hogates bien constituídos, cuya madre es atendida en la Caja de Seguro Obrero por intermedio de la libreta de su marido.

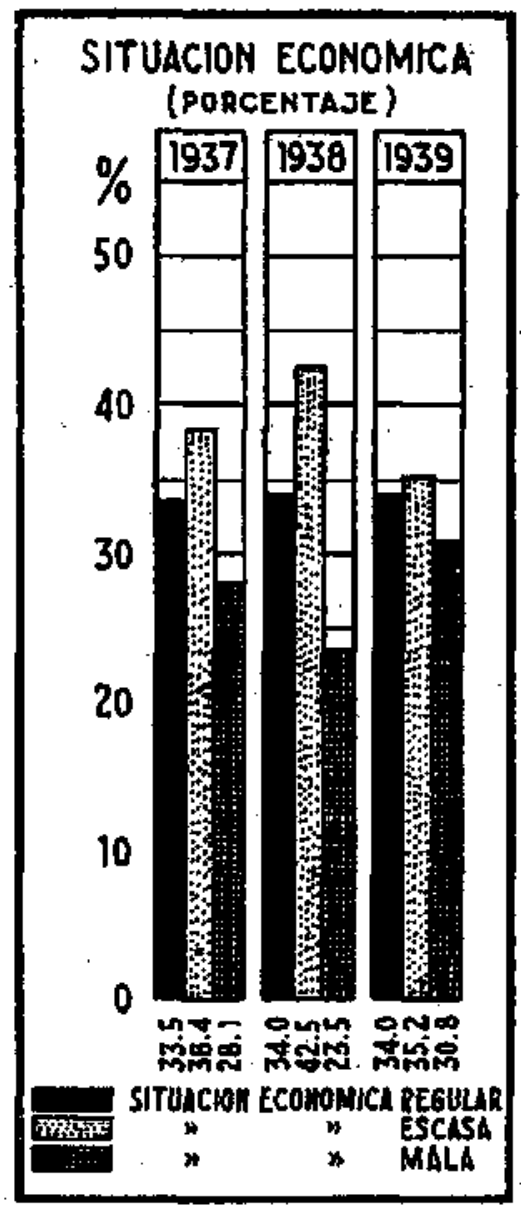

E1 Gráfico $N .^{9} 2$, que se refiere a la situación económica demuestra, en primer lugar, que el porcentaje de familias en mejor situación económica es casi igual al de años anteriores. $34 \%$. En estos bogares el término medio de las entradas dia- 


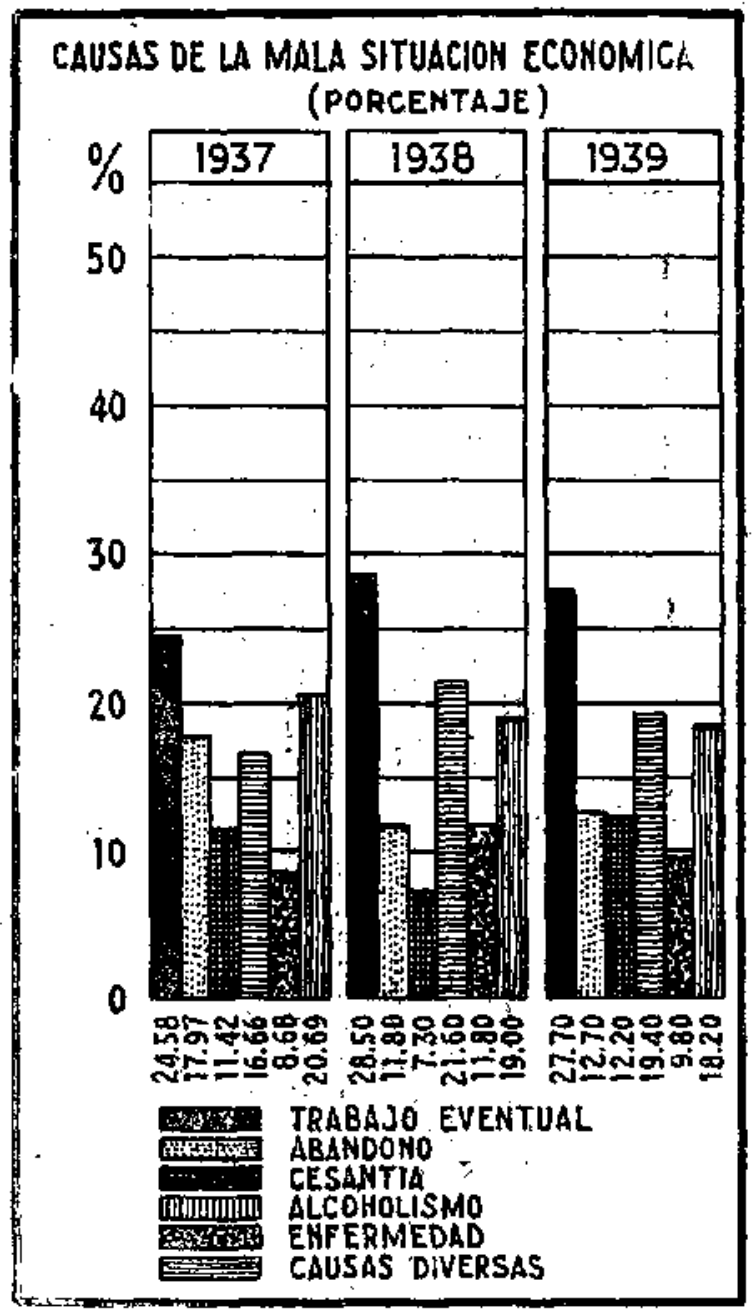

rias en cada uno de ellos es de $\$ 19,30$ y tienen un promedio de 2,6 hijos.

El segundo grupo, que hemos acordado llamar de situación económica escasa, tiene un porcentaje inferior, $35.2 \%$. Estas familias, dado el costo actual de la vida, "se mantienęn". pero no se alimentan, ya que es imposible alimentar a una familia con un promedio de 3,3 hijos y $\$ 11,80$ su entrada diaria. 


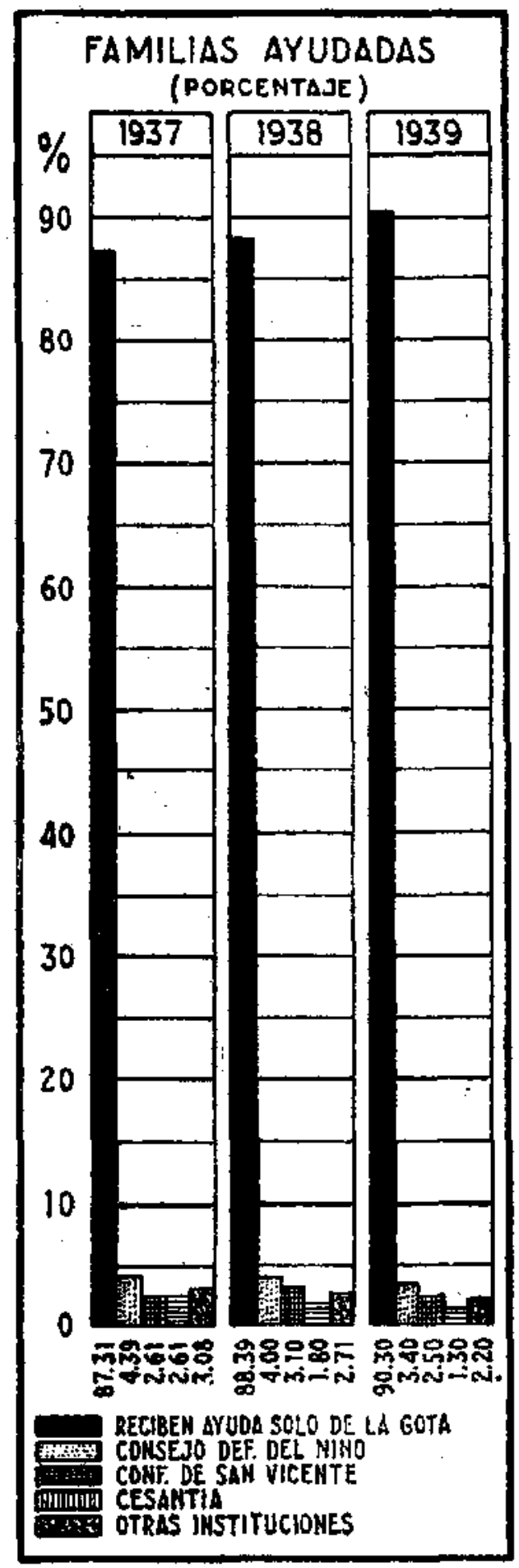




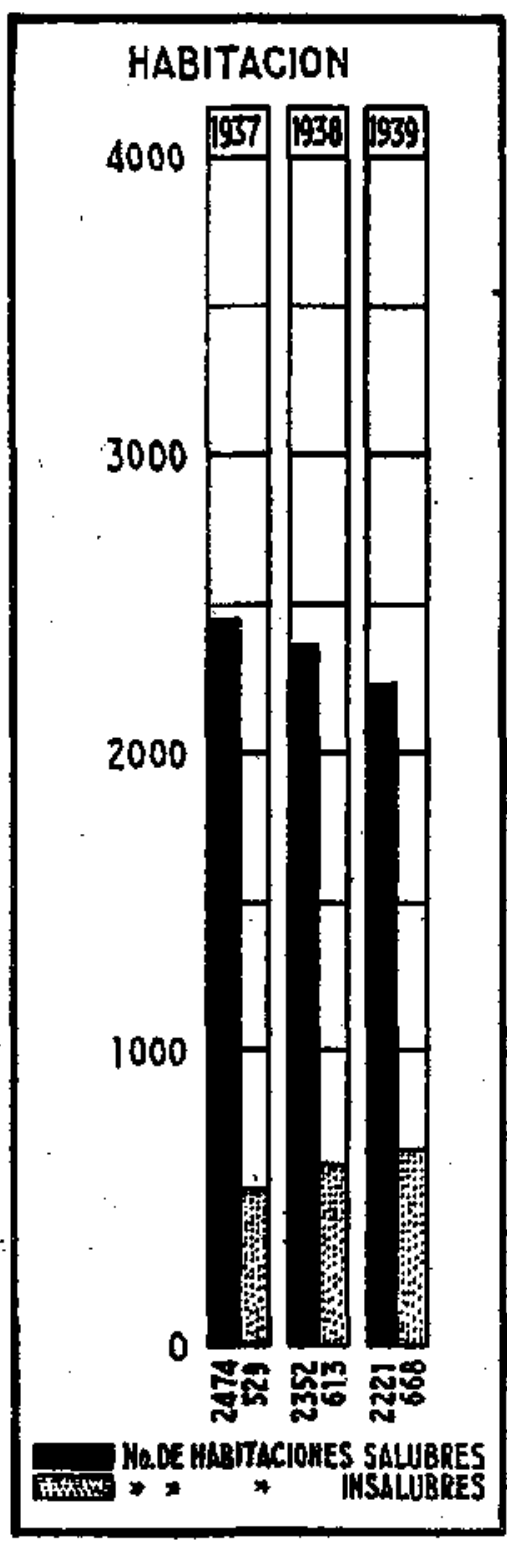

El tercer grupo lo constituye el $30,8 \%$ de nuestras familias, todas ellas indigentes y que viven en un estado de miseria y escasez económicos bastante grande, con un término medio de $\$ 6.70$ diarios $y$ un porcentaje de 3,5 hijos. 
Las causas de esta mala situación económica están analizadas en el Gráfico N.\% 3.

Hay que reconocer que gran parte de esta mala situación económica se debe a la falta de preparación de los obreros, a la mals inversión que dan al salario, al vicio, a la natural

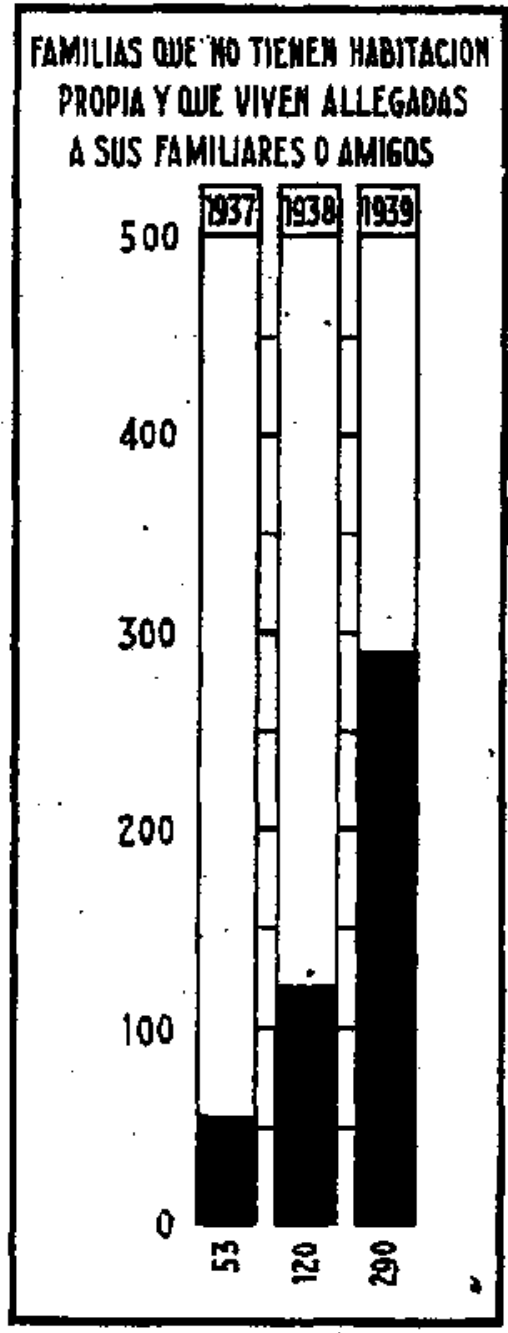

apatía del pueblo chileno, que sólo piensa en vivir al dia: sin ambiciones de surgir para llegar a conquístar para él y los suyos, un relativo bienestar. Por último, el alza del costo de 
la vida impide al obrero un mejoramiento de sus condiciones. aun cuando hoy día gana un salario un poco más alto que en años anteriores.

El Gráfico N. 5 corresponde a las habitaciones.

Por una habitación calificada salubre se paga un canon de arrendamiento de $\$ 65$ mensuales y viven 4,7 personas por pieza y 2,1 personas por cama. En las insalubres se paga como canon de arriendo un término medio de $\$ 38.10$ mensuales. Viven 5,6 personas por pieza y 2,8 personas por cama.

Hay 105 familias que mediante esfuerzos de largos años han conseguido obtener algún sitio donde han efectuado mejoras o cuentan con casítas que adquíeten mediante el pago de pequeñas cuotas mensuales.

Paralelo a este cuadro, exponemos el contraste: hay 290 familias, como lo demuestra el gráfico $N^{9} 6$ que viven allegadas a sus familias o amigos demostrando con ello uno de los problemas más graves de nuestro pueblo.

Al terminar la breve exposición de estos gráficos, que. remos hacer resaltar, someramente, los grandes problemas que aquejah al obrero chileno: males morales y económicos. Los unos derivados de los otros, repercutiendo todos en la colectividad. La ignorancia, la habitación, la promiscuidad, el alcoholismo, el salario insuficiente junto al alza de la vida, hacen que desarrolle sus actividades en un standard de vida bajo. A la acción gubernativa corresponde encarar estos problemas y las instituciones particulares secundar su labor.

Por ahora, nuestros esfuerzos son reducidos, dados los escasos recursos con que contamos y abarcan tanto la acción preventiva como la curativa. La labor desarrollada respecto a la educación dió algunos resultados, pues de 2,342 niños matriculados a principios del año 1939 en las diferentes escuelas, el $70.8 \%$ asistió regularmente a clases; las inasistencias restantes no fueron puestas en conocimiento de la Visitadora Social de la Gota de Léche en su debida oportunidad. Los escolares inscritos contaron con el vestuario indispensable. pues las Directoras de mestros.Servicios, previo informe de la Visitadora Social respectiva, proporcionaron lo necesatio a aquéllos que no habían sido matriculados por su indigencia. A simple vista resalta aquí la necesidad de la colaboración de la Escuela con el Servicio Social, o. más bien, que aquélla cuente con un Servicio Social propio.

El alcoholismo, corolario lógico de la deficiente educación que se proporciona al individuo desde niño, es la calusa de la mala situación económica en $19,40 \%$ de los hogares. 
La obra de la Visitadora Social es en estos casos pasiva, ya. que se necesita una serie de factores que no pueden reblizar; sólo se limita a remediar èn parte las consecuencias del vicio del padre, como ser: miseria, inmoralidad, enfermedad, etc.

Traslados.-Esta Inspección estima que, para facilitar la perfecta atención de la familia, debe ésta vivir dentro del radio correspondiente a cada Gota de Leche a fin de que su atención no sufra interrupciones como ha sucedido en más de alguna oportunidad. Es recesario insistir ante el personal acerca de la obligación que tiene de cumplir el reglamento en 10 que se refiere a traslados; esto significa simplificar la labor. lo que redunda en una mejor atención de nuestros niños.

"Día dz ila Gota de Leche".-Creemos que es necesario fomentar en cada barrio el cariño por la Gota de Leche, bus. cando la cooperación de las Instituciones y vecinos más caracterizados. Con este objeto, el Directorio dió su aprobación a lo propuesto por la Comisión de Servicio Social en el sen, tido de reemplazar la Semana de la Gota de Leché por el "Día de la Gota de Leche", a fin de que el personal atienda al público que visite los Servicios, le muestre la labor que se hace y la utilidad que podría tener su cooperación.

Datos estadísticos.--En el transcurso de este informe, hemos visto la labor desarrallada por las Visitadoras Sociales. Acompañamos a continuación sólo una parte del trabajo demostrado en datos estadisticos, ya que no es posible exponer todos los aspectos de los problemas sociales que comprenden tanto al individuo como a la colectividad en sus más variadas manifestaciones.

Visitas:

Domiciliarias por encuestas

Domiciliarias por casos pendientes

A Instituciones de Beneficencia

Legalizaciones:

Matrimonios civiles .............................. 194

Inscripción Registro Civil sin orden judicial ...... 156

Niños legitimados

Trámites Judiciales:

Nombramiento de curador 
Peticiones al Juzgado de Menores

Otras tramitaciones

Trámites Religiosos:

Matrimonios

Bautismos

Labor de orden económlica:

Benêficios conseguidos

Empleos

Donaciones

Préstamos

Imposiciones de Ahorro

$\$ 201$

Cooperación Médica:

Hospitalizaciones

Envios a Policlinicos para investigar diagnósticos

Vigilancia de tratamientos ambulatorios

Asistencia médica a domicilio

Envios fuera de Santiago por cambio de clima

Cambio de habitación por insalubre.

Ley 4045:

Reclamos incumplimiento de la Ley ............ .

Cobro de subsidios

Benefícios conseguidos

Otros casos sociales:

Colocaciones de niños en Instituciones de Protec. ción Cerrada

Niños en colocación familiar ............................. 68

Para terminar, diremos que el éxito obtenido por el Servicio Social se debe, en gran parte, al apoyo decidido del Dxrectorío del Patronato y de todos los que colaboran en esta obra de protección al niño. Los asistidos por las Gotas de Leche llegan generalmente sin amparo de ninguna especie: es el grupo más indigente de la sociedad, al que las leyes de previsión no alcanzan a proteger; pero que encuentran en el Pa. tronato lo que la vida les ha negado: atención desinteresada, técnica esmerada y lo que es más importante, un ambiente de cordialidad y abnegación para mitigar o solucionar sus problemas. El ambiente ampliamente humano y acogedor que brinda el Patronato a todos los que llegan a solicitar sus servicios. el anheio que cada uno de sus servidores pone en resolver los problemas sometidos a su iniciativa y la tradición honrosa que ha logrado formarse en nuestro pueblo, constituyen las bases más sólidas de su prestigio, y por eso no es extrañe que acudan familias que podrian obtener mayores beneficios en otras Instituciones. 